1 Evolutionary study of the $412 / m d g 1$ lineage of the Ty3/gypsy group of LTR

retrotransposons in Diptera

\section{Evolution of the 412/mdg1 lineage of the Ty3/gypsy group of LTR retrotransposons in Diptera}

Natasha Avila Bertocchi ${ }^{1}$, Fabiano Pimentel Torres ${ }^{2}$, Maríndia Deprá ${ }^{1}$ and Vera Lúcia da Silva Valente ${ }^{1^{*}}$

1Programa de Pós-Graduação em Genética e Biologia Molecular, Universidade Federal do Rio Grande do Sul, Porto Alegre, Rio Grande do Sul, Brazil

2Laboratório de Diversidade Genética Animal, Universidade Federal do Pampa, Campus São Gabriel, Rio Grande do Sul, Brazil

${ }^{*}$ Corresponding author

Email: vera.gaiesky@ufrgs.br (VSL)

\title{
Abstract
}

LTR-retrotransposons are structurally similar to retroviruses, as they possess the enzymes reverse transcriptase, Ribonuclease $H$, integrase, proteinase, and the gag gene and are flanked by long terminal repeats (LTRs). The 412/mdg1 lineage, belonging to the Ty3/Gypsy group, consists of the TEs 412, mdg1, stalker, pilgrim, and blood. The $412 / m d g 1$ lineage is distinguished from the others in the gypsy group in that it has small ORFs at the beginning of the TE and is highly similar to the pol ORF among the TEs that make up the 
lineage. In this study, our aim was to elucidate the evolutionary history of the $412 / m d g 1$ lineage in the 127 dipteran genomes available to date, and the characteristics of the sequences in each genome. We used the canonical TE 412 probe described in Drosophila melanogaster as the query. We found sequences homologous to the $412 / m d g 1$ lineage restricted to the suborder Brachycera. These sequences are widely distributed in drosophilids but are also present in other groups of flies. We note the presence of the $412 / \mathrm{mg} 1$ lineage in tsetse flies (Glossina). Furthermore, our results showed an elaborate evolutionary history for the $\mathrm{pol}$ ORF in the $412 / m d g 1$ lineage of the LTRRetrotransposon.

Keywords: Drosophila, Glossina, transposable elements

\section{Introduction}

Transposable elements (TEs) are part of the repetitive fraction of the genome capable of mobilizing within and between genomes. These characteristics of mobilization and parasites in genomes make TEs the most abundant and ubiquitous sequences in nature, influencing the evolutionary trajectory of host genomes in different ways [1]. TEs may be involved in diseases such as retrotransposon $L 1$ in the development of cancers in humans [2]; in chromosomal changes such as the Galileo element, which causes chromosomal inversions in Drosophila buzzatii [3]; or even in influencing the expression of genes, such as the accord-like element in the $5^{\prime}$ region of cytochrome P450, which confers insecticide resistance in D. melanogaster [4]. TEs also influence the size of the genomes and constitute the largest fraction of 
eukaryotic genomes. TEs may comprise $65 \%$ of the genome of insects, as in the migratory locust (Locusta migratoria) agricultural pest in Africa [5].

TEs can be divided according to the mechanism and intermediate transposition molecule [6-8]. Retrotransposons have the system called 'copyand-paste' and RNA as an intermediate mobilization, and DNA transposons with the 'cut-and-paste' system and DNA as an intermediate molecule for transposition. Retrotransposons can be subdivided into two large groups: retrotransposons without long terminal repetitions (LTRs), and retrotransposons with LTRs. Both groups of retrotransposons share their evolutionary history with viruses, but only LTR-retrotransposons share an evolutionary history with retroviruses [9].

Retroviruses, endogenous retroviruses (ERVs), and retrotransposons have a common origin. All three must be transcribed back into DNA to insert/excise in the host genome, for which they use a similar enzyme structure. Retroviruses infect vertebrates and if there is integration with the host's chromosomes in the germ cells allowing them to perpetuate in the species, they become the so-called endogenous retroviruses (ERVs) [10]. In the genomes of non-vertebrate eukaryotic organisms, retrovirus-like are called LTRretrotransposons [9].

LTR retrotransposons have similar characteristics to retroviruses, such as LTRs flanking the open reading frames (ORFs) that code for proteinase (PR), reverse transcriptase (RT), Ribonuclease $H(R H)$, integrase (INT), and gag-like proteins; and are organized similarly to retroviruses [11,12]. However, only a few LTR retrotransposons have putative env genes, responsible for the 
viral envelope [9]. The gag gene (ORF1) is responsible for the virus-like particle (VLP), and the pol gene (ORF2) is responsible for and the other enzymes (PR$\mathrm{RT}$-RH-INT) that are responsible for the mobilization. LTR-retrotransposons are mobilized via a mechanism that requires reverse transcription of mRNA. The $5^{\prime}$ LTR has a promoter that is recognized by the host's RNA pol II, after transcription of the intermediate mRNA, which is exported to the cytoplasm where the translation occurs. The VLP encapsulates the mRNA, which is reverse-transcribed, giving rise to the cDNA. The latter is then sent to the nucleus, where it will be inserted into the host genome by the action of integrase [13].

The phylogenetic relationships of LTR-retrotransposons subdivide them into two groups, called Ty1/copia and Ty3/Gypsy [14]. The phylogenetic tree of the Ty3/Gypsy group has two large branches, based on the pol ORF (PR-RTRH-INT) [15]. The first branch, called chromoviruses because they contain a chromodomain in the integrase, consists of TEs of plants, fungi, and vertebrate animals [14-16]. The second branch, called non-chromoviral, consists of TEs of insects and other groups of organisms $[14,15,17,18]$. Inside the Ty3/Gypsy nonchromoviral branch is the $412 / m d g 1$ lineage [17].

The $412 /$ mdg1 lineage consists of the 412, stalker, pilgrim, blood, and mdg1 elements, characterized in the genome of D. melanogaster [17]. Retrotransposon 412 was the first of the $412 / m d g 1$ lineage to be described, with approximately 7000 base pairs (bp) $[19,20]$. Retrotransposons of the $412 /$ mdg1 lineage share structural features such as two small ORFs (sORFs) within the $5^{\prime}$ region, ORF1 (gag), and ORF2 $(p o l)[14,17,19]$. Putative sORFs are a 
distinctive feature of the $412 / m d g 1$ lineage, but still have no known role in the survival of the element [21,22]. However, copies of blood without sORFs showed greater competitive potential compared to copies with sORFs in natural populations of $D$. melanogaster [22]. In addition, when analyzed separately, the sORFs, ORF1 and ORF2, showed differences in phylogenetic relationships between the elements of the lineage [17]. However, the same phylogenetic relationships among the elements of the lineage were observed in the alignment of each of the different domains (PR-RT-RH-INT) of ORF2 [17].

Historically, the reconstruction of the phylogenetic relationships of LTRretrotransposons is based on the RT domain, as it is one of the largest and most conserved domains. These evolutionary relationships were later supported with the addition of the other ORF2 domains $[9,15,23-26]$. In addition, the RT and $\mathrm{RH}$ domains have been shown to provide a good resolution of the phylogenetic relationships within the Ty3/gypsy group [24].

Within Insecta, the order Diptera is the best characterized in relation to the fraction of transposable elements (TEs); however, most studies have examined model organisms such as species of the genus Drosophila or mosquito disease vectors such as Aedes aegypti $[27,28]$. The percentage of TEs varies widely among dipteran genera, ranging from $1 \%$ in the fly Belgica antarctica (restricted to Antarctica) to more than $50 \%$ in Aedes aegypti $[27,29]$. Within the TEs, the order of retrotransposons constitutes the largest fraction of TEs in dipteran genera $[27,28,30]$. Our research group has been studying the presence of TEs, including 412 in Neotropical species of Drosophila, where we supposed a priori that the wide diversity of biomes and environments may have 
provided many opportunities for evolutionary adjustments in these genomes.

Blauth et al. [31], comparatively analyzing 412 of $D$. melanogaster and $D$. willistoni, observed that $D$. willistoni showed more restricted hybridization patterns during embryogenesis, probably due to the difference in sequence similarity between the canonical TE of $D$. melanogaster and the TE in the $D$. willistoni genome. The present study aimed to extend the investigation of the TEs of Neotropical species and/or other native or introduced species of flies, exploiting newly available methods.

The increase in genome sequencing provides an extraordinary opportunity to deepen knowledge about the evolution of TE lineages such as $412 / m d g 1$, and to fill the gaps in the distribution and phylogeny of their main lineages in the dipteran genomes. In this study, we peovie evidenve the conservation of the enzymes that constitute ORF2 in the $412 / m d g 1$ lineage in the genomes of different evolutionary lines of flies. We have possibly identified

a new branch in the $412 /$ mdg1 lineages in the species of the genus Glossina (tsetse), the vector of the parasite that causes human sleeping sickness. Furthermore, we elucidated the intricate nature of the evolutionary relationships involving the ORF2 sequences of the $412 / m d g 1$ lineage.

Material and Methods

Identification of $\mathbf{4 1 2}$ sequences from dipteran genomes 

available from NCBI.

Table 1: Presence/absence of 412-like sequences in the genomes of Order

Diptera. (+) 412-like elements present; (-) 412 -like elements absent.

\begin{tabular}{|c|c|c|c|c|c|}
\hline Order & Family & Subfamily & Genus & Subgenus & Grol \\
\hline \multirow[t]{32}{*}{ Brachycera } & Agromyzidae & Phytomyzinae & Liriomyza & - & - \\
\hline & Asilidae & Asilinae & \multicolumn{2}{|l|}{ Proctacanthus } & \\
\hline & & Trigonomiminae & Holcocephala & - & - \\
\hline & Glossinidae & - & Glossina & Fusca & - \\
\hline & & - & & Morsitans & - \\
\hline & & - & & & - \\
\hline & & - & & & - \\
\hline & & - & & Palpalis & - \\
\hline & & - & & & - \\
\hline & Calliphoridae & Calliphorinae & Calliphora & - & - \\
\hline & & Chrysomyinae & Cochliomyia & - & - \\
\hline & & & Phormia & - & - \\
\hline & & Luciliinae & Lucilia & - & - \\
\hline & & & & - & - \\
\hline & Diopsidae & - & Sphyracephala & - & - \\
\hline & & - & Teleopsis & - & - \\
\hline & Dolichopodidae & Sciapodinae & Condylostylus & - & - \\
\hline & Drosophilidae & Drosophilinae & Drosophila & Dorsilopha & $b u$ \\
\hline & & & & Drosophila & \\
\hline & & & & & \\
\hline & & & & & \\
\hline & & & & & $m \epsilon$ \\
\hline & & & & & \\
\hline & & & & & \\
\hline & & & & & quin \\
\hline & & & & & repl \\
\hline & & & & & \\
\hline & & & & & \\
\hline & & & & & \\
\hline & & & & & \\
\hline & & & & & $1010 \mathrm{c}$ \\
\hline & & & & & \\
\hline
\end{tabular}


bioRxiv preprint doi: https://doi. org/10.1101/2020.09.24.311225. this version posted September 24,2020 . The copyright holder for this preprint (which was not certified by peer review) is the author/funder, who has granted bioRxiv a license to display the preprint in perpetuity. It is made available under aCC-BY 4.0 International license.

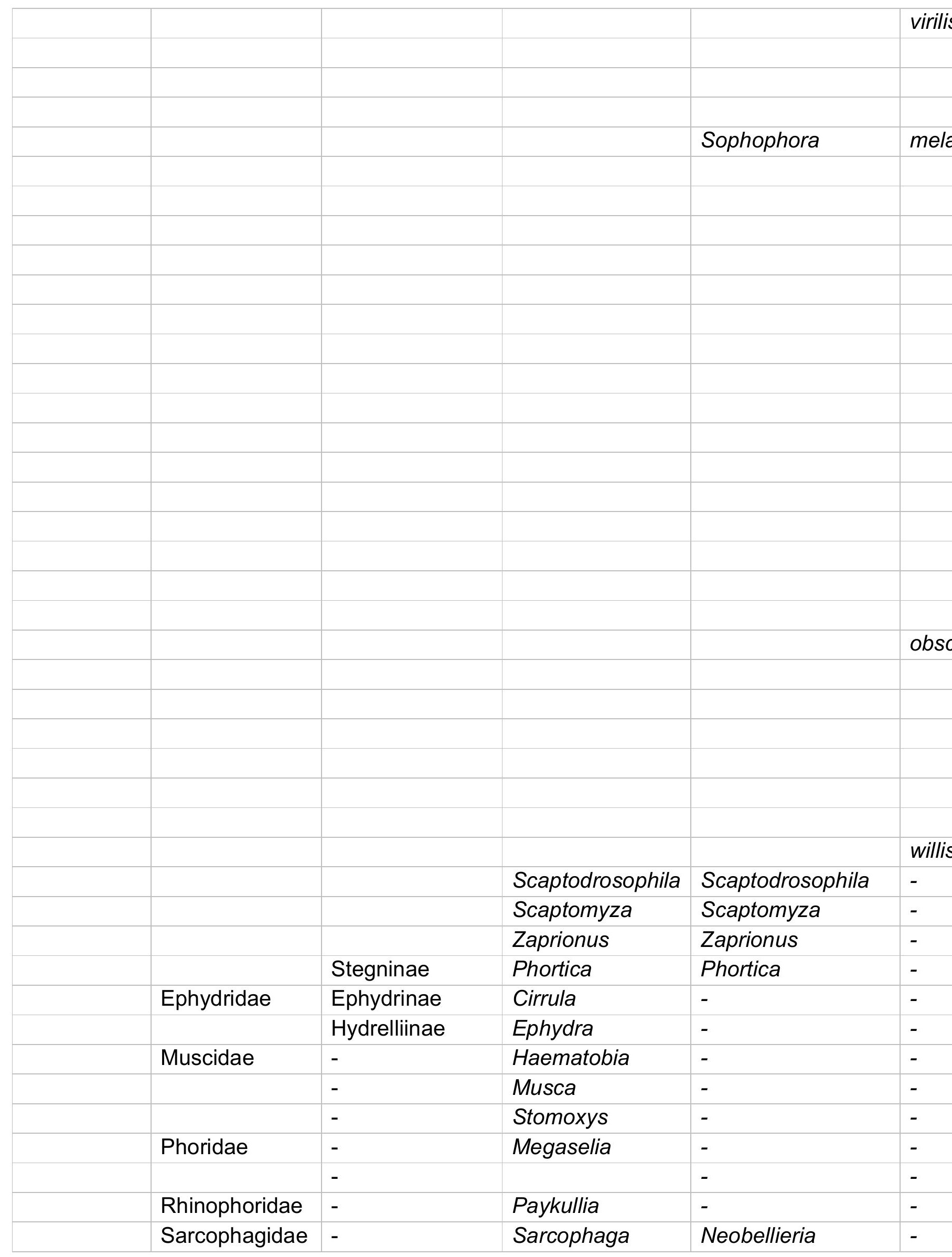




\begin{tabular}{|c|c|c|c|c|c|}
\hline & Sepsidae & - & Themira & - & - \\
\hline & Stratiomyidae & Hermetiinae & Hermetia & - & - \\
\hline & Syrphidae & Eristalinae & Eristalis & - & - \\
\hline & Tephritidae & - & Bactrocera & Bactrocera & - \\
\hline & & - & & & - \\
\hline & & - & & & - \\
\hline & & - & & Daculus & - \\
\hline & & - & Ceratitis & Ceratitis & - \\
\hline & & - & Eutreta & Metatephritis & - \\
\hline & & - & Rhagoletis & - & - \\
\hline & & - & Tephritis & - & - \\
\hline & & - & Trupanea & . & . \\
\hline & & - & Zeugodacus & Zeugodacus & - \\
\hline \multirow[t]{26}{*}{ Nematocera } & Cecidomyiidae & Cecidomyiinae & Mayetiola & - & - \\
\hline & Chaoboridae & - & Chaoborus & - & - \\
\hline & & - & Mochlonyx & - & - \\
\hline & Chironomidae & - & Belgica & - & - \\
\hline & & - & Clunio & - & - \\
\hline & & Chironominae & Chironomus & - & - \\
\hline & & & & Camptochironomus & - \\
\hline & & & Culicoides & Monoculicoides & - \\
\hline & Culicidae & Anophelinae & Anopheles & - & - \\
\hline & & & & - & - \\
\hline & & & & - & - \\
\hline & & & & Anopheles & - \\
\hline & & & & & - \\
\hline & & & & Cellia & - \\
\hline & & & & & - \\
\hline & & & & & - \\
\hline & & & & & - \\
\hline & & & & & - \\
\hline & & & & & - \\
\hline & & & & & - \\
\hline & & & & & - \\
\hline & & & & & - \\
\hline & & & & & - \\
\hline & & & & & - \\
\hline & & & & & - \\
\hline & & & & & - \\
\hline
\end{tabular}




\begin{tabular}{|c|c|c|c|c|}
\hline & & & & - \\
\hline & & & & - \\
\hline & & & & - \\
\hline & & & Nyssorhynchus & - \\
\hline & & & & - \\
\hline & & & & - \\
\hline & Culicinae & Aedes & Stegomyia & - \\
\hline & & & & - \\
\hline & & Culex & Culex & - \\
\hline Psychodidae & - & Phlebotomus & Phlebotomus & - \\
\hline & Phlebotominae & Lutzomyia & Lutzomyia & - \\
\hline & Psychodinae & Clogmia & - & - \\
\hline Scatopsidae & - & Coboldia & - & - \\
\hline Tipulidae & - & Tipula & Tipula & - \\
\hline
\end{tabular}
in the NCBI database [32]. A list of the species analyzed and the corresponding sequence data is provided in Table 1. The sequences with an E-value lower 150 than e-10 were extracted. In Table 1: column "BLAST_412 query" are the genomes with positive results in BLASTn, and column "412-like" are the genomes with homologous sequences after refinement (described below).

\section{Sequence analysis}

The genome definition with the presence of sequences homologous to 412 was carried out with two stages of refinement. In the first stage, the homologous sequences in each genome were aligned using the MAFFT software with the default parameters, adjusting the direction according to the complete TE 412 reference sequence [33]. In this first stage, sequences that covered most of the element (412 query) or different segments of the TE were retained, making it possible to establish a consensus sequence for each genome. The consensus sequences were obtained using the UGENE software, 
162

with the default parameters showing the most common nucleotide in each position [34]. In the second stage, the previously generated consensus sequences were aligned again by the MAFFT software, using the G-INS-1 method and the direction of the reference sequence [33]. The alignments were refined manually when necessary and the sequences were translated using the Aliview software. Genomes containing 412-like elements were considered to be those that contained sequences with at least $50 \%$ similarity at the level of nucleotides and amino acids with the sequence of the internal region of the 412 element (X04132) (S1 Table). These are sORFs, the gag gene (ORF1), and the pol gene (ORF2), as originally described [19].

\section{Phylogenetic analyses}

Conserved ORF2 domains (RT-RH) were used in the phylogenetic analyses as described by [25]. The domains were identified using the Conserved

Domain

Database

$(\mathrm{CDD})$

platform

https://www.ncbi.nlm.nih.gov/Structure/cdd/wrpsb.cgi. To confirm the relationship of the sequences obtained with the elements of the $412 / m d g 1$ lineage, the following $D$. melanogaster sequences were included in the analysis: 412 (GenBank accession number X04132), mdg1 (GenBank accession number X59545.1), pilgrim (GenBank accession number AC007146), and STALKER4_I and BLOOD_I from the Repbase database. Element 17.6 (GenBank number X01472.1) was used as the outgroup. Bayesian phylogenetic analysis was performed with BEAST v1.10.4 software, using the LG model [35] with gamma distribution and invariant sites, through Cipres Computational Resources [36]. 
185

The Markov Chain Monte Carlo (MCMC) was run for 10,000,000 iterations, with trees saved every 10,000 iterations, after a 2,500 burn-in.

The phylogenetic reconstruction of the analyzed species was based on the nuclear gene Amyrel. Sequences were downloaded using the access number provided by Tambones et al. [37] (S2 Table). Additional sequences were obtained directly from $\mathrm{NCBI}$, using $D$. melanogaster as a query or another related species when no results were recovered. Bayesian inference was performed with BEAST v1.10.4 software, using General Time Reversible (GTR) with gamma distribution and invariant sites, through Cipres Computational Resources [36]. The mosquito species Anopheles gambiae was used as the outgroup.

\section{Median-joining networks}

Diagrams using Median-joining were obtained from conserved regions used in previous phylogenetic reconstructions (conserved domains RT-RH). The alignments were converted to the Roehl format, with deletion of the invariant sites using the DNASP version 6 program, and the diagrams were generated using the Fluxus Network software, with default parameters $[38,39]$.

\section{Results}

\section{Genomes with 412-like retrotransposon}

The presence of 412 -like sequences was evaluated in 127 available genomes of the order Diptera: 87 genomes of suborder Brachycera (flies) and 40 genomes of suborder Nematocera (mosquitoes) (Table 1). Initially, the 
208

209

210

211

212

preliminary results identified 412-like sequences in 65 genomes, 61 in Brachycera and only 4 in Nematocera (Table 1). However, the sequences from species of Nematocera and some species of Brachycera, after refinement, proved to be quite divergent from the 412-like sequences and were excluded (Table 1 and S1 Table).

Within the suborder Brachycera, sequences were identified in the families Tephritidae (1 species), Glossinidae (5 species), Muscidae (1 species), Diopsidae (1 species) and Drosophilidae (36 species). Thus, the 412-like sequences showed a wide distribution in the subfamily Drosophilinae, including the genera Scaptodrosophila, Scaptomyza, and mainly Drosophila (Table 1).

\section{Sequences homologous to lineage $412 /$ mdg1}

The complete sequence of the internal region (sORFs, ORF1, and ORF2) of 412-like were detected only in the Drosophila genome, in $D$. melanogaster, D. mauritiana, and D. simulans (Fig 1). In D. sechellia a complete sequence of 412 was not identified and all copies had some deletions; however, it was possible to obtain a consensus sequence from an assembly of these sequences (Fig 1). The copies detected in these genomes were identical or nearly so to the reference 412 (accession number X04132) at the nucleotide level, ranging from $94 \%$ in D. sechellia to $100 \%$ in D. melanogaster (S3 Table).

Figure 1: Schematic representation of the reconstructed 412-like copies compared to the $\mathbf{4 1 2}$ canonical. The gray rectangles indicate an untranslated leader region (ULR) and two small putative ORFs (sORFs), the gag gene (ORF1) and the pol gene (ORF2). Different domains of the ORF2 are indicated as follows: protease, PR; 
232

reverse transcriptase, $\mathrm{RT}$; Ribonuclease $\mathrm{H}, \mathrm{RH}$; integrase, INT. Species were divided into four groups according to their structural characteristics. Groups I and II are Family Drosophilidae, subgenus Sophophora - melanogaster group. I - 412 (X04132), Drosophila melanogaster, D. mauritiana, D. sechellia, D. simulans. II - D. erecta and $D$. yakuba. III - Subgenus Drosophila - virilis group: D. virilis; IV - Family Glossinidae, subgenus Morsitans: Glossina austeni, G. morsitans, G. pallidipes; Subgenus Palpalis: G. fuscipes, G. palpalis; Family Diopsidae: Teleopsis dalmanni; Family Drosophilidae, subgenus Drosophila - melanica group: D. melanica, D. micromelanica, $D$. nigromelanica, D. innubila; repleta group: $D$. hydei, $D$. mojavensis; robusta group: $D$. lacertosa, D. robusta; virilis group: D. americana, D. montana, D. novamexicana; Subgenus Sophophora - melanogaster group: D. ananassae, D. biarmipes, $D$. bipectinata, D. elegans, D. eugracilis, D. ficusphila, D. kikkawai, D. rhopaloa, D. serrata, D. suzukii, D. takahashi; obscura group: D. miranda, D. obscura, D. persimilis, D. pseudoobscura; willistoni group: D. willistoni; Subgenus Scaptodrosophila: Scaptodrosophila lebanonensis; Subgenus Scaptomyza: Scaptomyza flava; Family Muscidae: Musca domestica; Family Tephritidae: Rhagoletis zephyria

Other regions of the TE in the remaining genomes, analyzed only in the species D. erecta, D. yakuba, D. virilis, D. suzukii, and Scaptomyza lebanonensis, possess sequences partially homologous to ORF1 of 412 . In addition, all genomes analyzed showed sequences homologous to ORF2 of 412. The similarity of the sequences in relation to the query, level of amino acids, ranged between $100 \%$ in D. melanogaster and $53 \%$ in D. nigromelanica (Fig 1 and S1 Table). Among the enzyme genes that constitute ORF2, the most conserved sequences were related to $\mathrm{RT}$ and $\mathrm{RH}$.

\section{Evolutionary analysis of the sequences evaluated}


The searches for the dispersion of 412 in the dipteran genomes showed

258

that the elements of the $412 / m d g 1$ lineage are very similar to each other, as seen in Figures 2 and 3 . We reconstructed the phylogenetic relationships of the $412 / m d g 1$ lineages based on a fragment of ORF2, the conserved regions of the $\mathrm{RT}$, and RH genes from the consensus sequences obtained. The consensus sequences represent an approximation of the genes that gave rise to the visible copies in the genomes. The resulting tree showed a topology with the species of flies, with emphasis on Drosophila, not grouping according to its host genome, as observed in the phylogeny of the Amyrel gene (S1 Fig). Musca domestica, R. zephyria, and T. dalmani were positioned in more external branches (Fig 2). Lineages 412, stalker, and pilgrim formed three distinct arms, where most species were grouped (Fig 2). However, the lineages $m d g 1$ and blood were placed in another outer arm, distant from the other lineages and shared with the Glossina sequence.

\section{Figure 2: Phylogenetic reconstructions of the $412 / m d g 1$ lineage based on} reverse transcriptase $(R T)$ and Ribonuclease $H(R H)$ regions. Bayesian phylogenetic analysis was performed with BEAST v1.10.4 software [Cipres Computational Resources [36]], using the $L G+G+I$ model. The Markov Chain Monte Carlo (MCMC) was run for 10,000,000 iterations, with trees saved every 10,000 iterations, after a 2,500 burn-in. Lineage 412/mdg1: 412 (blue), stalker (pink), mdg1 (yellow), pilgrim (green), blood (orange), Glossina species (purple), and median vectors (red). Element 17.6 was used as the outgroup.

Figure 3: Network reconstructions. Median-joining network analyses for the $412 / m d g 1$ lineage. The size of each circle indicates the number of sequences 
281

282

grouped together. (a) Network for the elements in the $412 / m d g 1$ lineage. Each element in the lineage is differently colored. (b) The network of all sequences used in the phylogenetic reconstructions. (c) Detail of the $412 / m d g 1$ lineage relationships. The sequences are colored according to the clustering in the phylogenetic tree.

We built a network with the same consensus sequences used in the phylogenetic tree, as a new approach to analyzing the complex relationship between the different elements that comprised the mdg1/412 lineage and consequently shaped its evolution in different genomes. Inspection of the network shows that the elements 412 , stalker, and pilgrim are more closely related to each other than to $m d g 1$ and blood (Fig $3 A$ ). Analysis of all the sequences reveals the intricate relationship among the sequences, demonstrated by the central median vectors, which form an intricate network that links all the sequences (Fig 3B-C). On the other hand, 412 and stalker are not closely related to the phylogenetic tree but are inside sister groups.

The different approaches used to unveil the evolution of this group of TEs, phylogenetic tree and network, indicated that the sequences obtained in the five genomes that showed positive results in the BLAST of members of the genus Glossina formed a new group within the lineage 412/mdg1 (Figs 2 and $3)$.

\section{Discussion}

Our results showed that the sequences homologous to 412 in the order Diptera were restricted to the suborder Brachycera, which is composed of flies (Table 1). Dipterans diverged more than 350 million years ago (mya), and at 
305

306

307

approximately 300 mya divided between flies (Brachycera) and mosquitoes (Nematocera) [40]. Therefore, we have three possibilities for the elements of the 412/mdg1 lineage: 1) they remained in the fly branch; 2) they were lost in the mosquito group; 3 ) or they were inserted into the fly genome after the group diverged at approximately 300 mya.

In suborder Brachycera, the 412-like elements are widely distributed in the available genomes (Fig 1). However, complete sequences of 412 were available only for Drosophila melanogaster, D. mauritiana, $D$. simulans and $D$. sechellia (consensus) (Fig 1). We found complete sequences of 412, including integral LTRs (data not shown), only in closely evolutionarily related Drosophila species. D. melanogaster appeared between 2.5 and $3.4 \mathrm{Mya}$, and $D$. simulans, $D$. sechellia, and D. mauritiana diverged at approximately $0.5 \mathrm{Mya}$, forming the melanogaster supercomplex [41]. Furthermore, we also detected partially complete 412 copies in the genomes of $D$. yakuba, $D$. erecta, and $D$. ananasse, in the first two copies was missing the region of the sORFs, and in the last one, fragments of ORF2 were detected (Fig 1). Dias et al. [42] also identified complete sequences of 412 in these same species, except for $D$. mauritiana (did not analyze), and in addition to these were also identified in $D$. yakuba, $D$. erecta and $D$. ananasse.

In the phylogenetic tree, the members of the families Muscidae, Tephritidae, Diopsidae, Drosophilidae, and Glossinidae are divided into different branches (Fig 2). However, inspection of the internal branches shows that elements 412, stalker, and pilgrim form groups with different species regardless of the host genome (Figs. 2 and 4). The discrepancies between host genomes 
and the elements are more evident in the elaborate network formed by the 412/mdg1 lineage (Fig 3 A-C). Costas et al. [17], characterizing the 412/mdg1 lineage, observed differences in the phylogenetic relationships between the elements when the ORF1 and ORF2 were analyzed separately; these differences can be explained by the independent evolution of each of the conserved domains of the TEs [43]. However, did not identify differences in the relationships of the elements when analyzing the ORF2 domains individually [17].

The intricate relationships between the different elements of the $412 / m d g 1$ lineage, with the central median vectors forming an elaborate network (Fig 3C), probably occurred due to mosaicisms of these retrotransposons. In the $412 / m d g 1$ lineage, mosaicisms were identified within the sORFs and ORF1 between 412 and stalker, 412, and mdg1 in $D$. melanogaster, D. simulans and D. yakuba populations [8,21]. According to Costas et al. [17], the stalker element is the result of recombination events in the ancestral family of what are currently the other elements of the lineage. LTR retrotransposons may be prone to genetic rearrangements (mosaicisms), as two viral genomes packaged in the same virus-like particle can recombine during the reverse-transcription step between RNA genomes [44].

Another interesting feature of our results was the homologous sequences of the $412 / m d g 1$ lineage in Glossina. Of the six species analyzed, G. palpalis, G. fuscipes, G. austeni, G. morsitans, G. pallidipes, and G. brevipalpis, we not found homologous sequences of the $412 / m d g 1$ lineage in G. brevipalpis (Table 1). Knowledge of the genomic characteristics and TEs in tsetse flies is still 
353

354

sparse and preliminary. However, the authors who sequenced and analyzed the genomes of these 6 tsetse species observed that G. brevipalpis is the most basal species in the group, with the fewest TEs ( 35\% of the total genome), practically without LTR-TEs, and large expansion of mariner-like elements [30]. Possibly the $412 / m d g 1$ lineage was not maintained in G. brevipalpis as in the other species of Glossina. In addition, the sequences identified in the Glossina genomes may be another element of the $412 / m d g 1$ lineage, possibly diversified from the blood and mdg1 sequences (Figs 2 and 3). Furthermore, we identified sequences homologous to lineage $412 / m d g 1$ in $D$. persimilis (Figs 1 and 2), which had not previously been identified [23]. Bargues and Lerat [23] initially searched the LTRs of the elements and later analyzed ORF2. In our analyses, we searched the genomes of Diptera for the internal regions of the element (sORFs, ORF1, ORF2). We identified relics of the $412 / m d g 1$ lineage in the $D$. persimilis genome (Figs 1 and 2), but this allowed us to group this species together with other drosophilas, specifically with a sister group of $D$. obscura (Fig 2).

The $412 / m d g 1$ lineage is widely dispersed in the genomes of flies, not only in the family Drosophilidae, and at different levels of conservation in the pol gene. Our search for 412 in the genomes of Diptera revealed an intricate relationship in the evolution of ORF2 between the elements 412, stalker, pilgrim, blood, and mdg1. This indicates the importance of mosaicism in the evolutionary history of the $412 / m d g 1$ lineage. 
375

Acknowledgements: We are grateful to M.Sc. Thays Duarte de Oliveira and M.Sc. Henrique Moreira for their valuable assistance with the figures and phylogenetic analyses.

\section{References}

1. Aziz RK, Breitbart M, Edwards RA. Transposases are the most abundant, most ubiquitous genes in nature. Nucleic Acids Res. 2010;38: 42074217. doi:10.1093/nar/gkq140

2. Scott EC, Devine SE. The Role of Somatic $L 1$ Retrotransposition in Human Cancers. Viruses. 2017;9: 1-19. doi:10.3390/v9060131

3. Delprat A, Negre B, Puig M, Ruiz A. The transposon Galileo generates natural chromosomal inversions in Drosophila by ectopic recombination. PLoS One. 2009;4: e7883. doi:10.1371/journal.pone.0007883

4. Catania F, Kauer MO, Daborn PJ, Yen JL, Ffrench-Constant RH, Schlötterer C. World-wide survey of an Accord insertion and its association with DDT resistance in Drosophila melanogaster. Mol Ecol. 2004;13: 2491-2504. doi:10.1111/j.1365-294X.2004.02263.x

5. Wang $X$, Fang $X$, Yang $P$, Jiang $X$, Jiang $F$, Zhao $D$, et al. The locust genome provides insight into swarm formation and long-distance flight. Nat Commun. 2014;5:2957. doi:10.1038/ncomms3957

6. Finnegan DJ. Eukaryotic transposable elements and genome evolution. Trends Genet. 1989;5: 103-107. doi:10.1016/0168-9525(89)90039-5 
397

7. Wicker T, Sabot F, Hua-Van A, Bennetzen JL, Capy P, Chalhoub B, et al. A unified classification system for eukaryotic transposable elements. Nat Rev Genet. 2007;8: 973-982. doi:10.1038/nrg2165-c4

8. Kapitonov V V, Jurka J. A universal classification of eukaryotic transposable elements implemented in Repbase. Nat Rev Genet. 2008;9: 411-412; author reply 414. doi:10.1038/nrg2165-c2

9. Eickbush TH, Jamburuthugoda VK. The diversity of retrotransposons and the properties of their reverse transcriptases. Virus Res. 2008;23: 1-24. doi:10.1038/jid.2014.371

10. Benachenhou F, Sperber GO, Bongcam-rudloff E, Andersson G, Boeke JD. Conserved structure and inferred evolutionary history of long terminal repeats (LTRs). Mob DNA. 2013;4. doi:10.1186/1759-8753-4-5

11. Mount SM, Rubin GM. Complete Nucleotide Sequence of the Drosophila Transposable Element Copia: Homology Between Copia and Retroviral Proteins. Mol Cel Biol. 1985;5: 1630-1638. doi:doi:10.1128/mcb.5.7.1630

12. Xiong Y, Eickbush TH. Similarity of Reverse Transcriptase-like Sequences of Viruses, Transposable Elements, and Mitochondrial Introns. Mol Biol Evol. 1988;5: 675-690. doi:10.1093/oxfordjournals.molbev.a040521

13. Levin HL, Moran J V. Dynamic interactions between transposable elements and their hosts. Nat Rev Genet. 2011;12: 615-627. doi:10.1038/nrg3030.Dynamic 
et al. The Gypsy Database (GyDB) of mobile genetic. Nucleic Acids Res. 2011;39: 70-74. doi:10.1093/nar/gkq1061

15. Llorens C, Muñoz-Pomer A, Bernad L, Botella H, Moya A. Network dynamics of eukaryotic LTR retroelements beyond phylogenetic trees. Biol Direct. 2009;4: 6-31. doi:10.1186/1745-6150-4-41

16. Marín I, Lloréns C. Ty3/Gypsy Retrotransposons: Description of New Arabidopsis thaliana Elements and Evolutionary Perspectives Derived from Comparative Genomic Data. Mol Biol Evol. 2000;17: 1040-1049. doi:10.1093/oxfordjournals.molbev.a026385

17. Costas J, Valadé E, Naveira H. Structural Features of the mdg1 Lineage of the Ty3/gypsy Group of LTR Retrotransposons Inferred from the Phylogenetic Analyses of Its Open Reading Frames. J Mol Biol. 2001;53: 165-171. doi:10.1007/s002390010206

18. Nefedova LN, Mannanova MM, Kim Al. Integration specificity of LTRretrotransposons and retroviruses in the Drosophila melanogaster genome. Virus Genes. 2011; 297-306. doi:10.1007/s11262-010-0566-4

19. Yuki S, Inouye S, Ishimaru S, Saigo K. Nucleotide sequence characterization of a Drosophila retrotransposon, 412. Eur J Biochem. 1986;158: 403-410. doi:10.1111/j.1432-1033.1986.tb09767.x

20. Rubin GM, Finnegan D j, Hogness DS. The Chromosomal Arrangement of Coding Sequences In a Family of Repeated Genes. Prog Nucleic Acid Res Mol Biol. 1977;19: 221-226. doi:10.1016/S0079-6603(08)60920-4 
of Drosophila 412 Retrotransposable Element Generated by

Recombination. Mol Biol Evol. 2004;22: 747-757.

doi:10.1093/molbev/msi060

22. Costas J, Valadé E, Naveira H. Amplification and phylogenetic

Drosophila. J Mol Evol. 2001;52: 342-350. doi:10.1007/s002390010164

23. Bargues N, Lerat E. Evolutionary history of LTR-retrotransposons among

20 Drosophila species. Mob DNA. 2017;8: 1-15. doi:10.1186/s13100-

017-0090-3

24. Malik HS. Evolution of Retrotransposable Elements Ribonuclease H evolution in retrotransposable elements. Cytogenet Genome Res. 2005;110: 392-401. doi:10.1159/000084971

25. Malik HS, Eickbush TH. Modular Evolution of the Integrase Domain in the Ty3/Gypsy Class of LTR Retrotransposons. J Virol. 1999;73: 5186-5190. doi:10.1128/JVI.73.6.5186-5190.1999

26. Malik HS, Eickbush TH. Phylogenetic Analysis of Ribonuclease H Domains Suggests a Late , Chimeric Origin of LTR Retrotransposable Elements and Retroviruses. Genome Res. 2001;11: 1187-1197. doi:10.1101/gr.185101.1

27. Petersen M, Armisén D, Gibbs RA, Hering L, Khila A, Mayer G, et al. Diversity and evolution of the transposable element repertoire in arthropods with particular reference to insects. BMC Evol Biol. 2019;19: 1-15. doi:10.1186/s12862-018-1324-9 
466

467

28. McCullers TJ, Steiniger M. Transposable elements in Drosophila. Mob Genet Elements. 2017;7: 1-18. doi:10.1080/2159256X.2017.1318201

29. Kelley JL, Peyton JT, Teets NM, Yee M, Johnston JS, Bustamante CD, et al. Compact genome of the Antarctic midge is likely. Nat Commun. 2014;5: 1-8. doi:10.1038/ncomms5611

30. Attardo GM, Abd-alla AMM, Acosta-serrano A, Allen JE, Bateta R, Benoit $\mathrm{JB}$, et al. Comparative genomic analysis of six Glossina genomes, vectors of African trypanosomes. Genome Biol. 2019;20: 1-31. doi:10.1186/s13059-019-1768-2

31. Blauth ML, Bruno RV, Abdelhay E, Valente VLS. Spatiotemporal transcription of the $P$ element and the 412 retrotransposon during embryogenesis of Drosophila melanogaster and D. Willistoni. Genet Mol Biol. 2011;34: 707-710. doi:10.1590/S1415-47572011005000047

32. Altschul SF, Gish W, Miller W, Myers EW, Lipman DJ. Basic Local Alignment Search Tool. Journal of Molecular Biology. 1990. pp. 403-410. doi:10.1016/S0022-2836(05)80360-2

33. Katoh K, Standley DM. MAFFT multiple sequence alignment software version 7: Improvements in performance and usability. Mol Biol Evol. 2013;30: 772-780. doi:10.1093/molbev/mst010

34. Okonechnikov K, Golosova O, Fursov M, Varlamov A, Vaskin Y, Efremov I, et al. Unipro UGENE: A unified bioinformatics toolkit. Bioinformatics. 2012;28: 1166-1167. doi:10.1093/bioinformatics/bts091 
36. Miller MA, Pfeiffer W, Schwartz T. Creating the CIPRES Science Gateway for Inference of Large Phylogenetic Trees. 2010 Gateway Computing Environments Workshop (GCE), New Orleans, LA. 2010. pp. 1-8. doi:10.1109/GCE.2010.5676129

37. Tambones IL, Haudry A, Simão MC, Carareto CMA. High frequency of horizontal transfer in Jockey families (LINE order) of drosophilids. Mob DNA. 2019;10: 1-15. doi:10.1186/s13100-019-0184-1

38. Rozas J, Ferrer-Mata A, Sánchez-DelBarrio JC, Guirao-Rico S, Librado P, Ramos-Onsins SE, et al. DnaSP 6: DNA Sequence Polymorphism Analysis of Large Data Sets. Mol Biol Evol. 2017;34: 3299-3302. doi:10.1093/molbev/msx248

40. Thomas GWC, Dohmen E, Hughes DST, Murali SC, Poelchau M, Glastad

39. Bandelt H-J, Forster P, Rohl A. Median-Joining Networks for Inferring Intraspecific Phylogenies. Mol Biol Evol. 1999;16: 37-48. doi:10.1093/oxfordjournals.molbev.a026036

41. Hey J, Kliman RM. Population Genetics and Phylogenetics of DNA 
invasion of transposable elements in Drosophila species. BMC Evol Biol.

43. Capy $\mathrm{P}$, Maisonhaute $\mathrm{C}$.

C. Acquisition/Loss of Modules: the Construction

Set of Transposable Elements. Russ J Genet. 2002;38: 594-601. doi:10.1023/A:1016027530962

44. Hu WS, Temin HM. Genetic consequences of packaging two RNA genomes in one retroviral particle: Pseudodiploidy and high rate of genetic recombination. Proc Natl Acad Sci U S A. 1990;87: 1556-1560. doi:10.1073/pnas.87.4.1556

\section{Supporting information}

S1 Fig: Phylogenetic relationships between sequences in the nuclear performed with BEAST v1.10.4 software, using $G T R+G+I$ as the substitution model, through Cipres Computational Resources [36]. Outgroup: Anopheles gambiae.

S1 Table: Distance matrix of 412 -like sequences. Nucleotide and amino acid

528 distance matrix of sequences 412-like, using UGENE software [34].

529 S2 Table: Acess number Amyrel gene.

S3 Table: Distance matrix of complete copies of 412 in Drosophila.

531 Distance matrix based on the nucleotide sequences of complete copies of 412 532 in Drosophila, using the UGENE software [34]. 

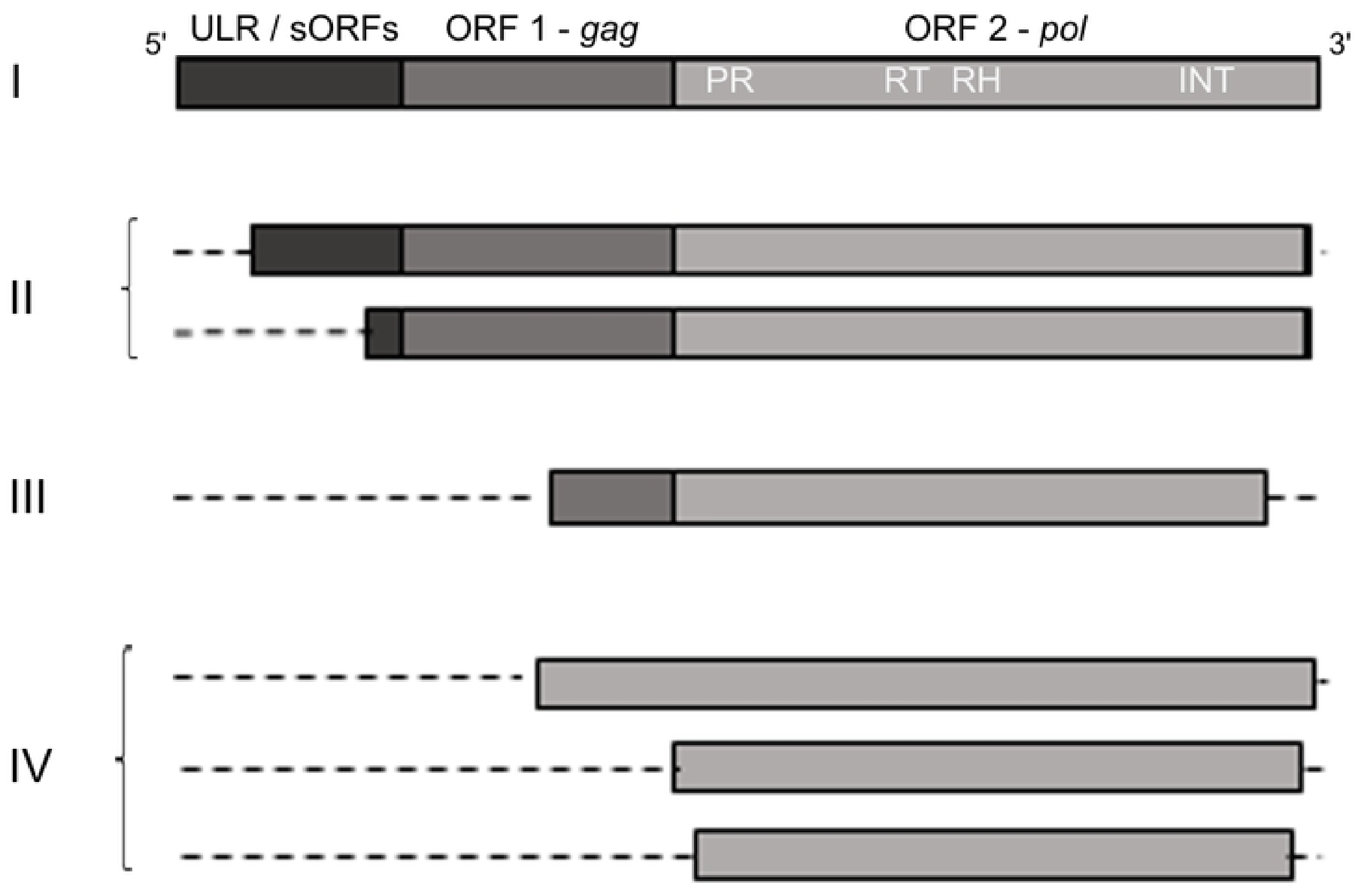

Figure 1 
A<smiles>O=C(O)C(O)O</smiles>

B

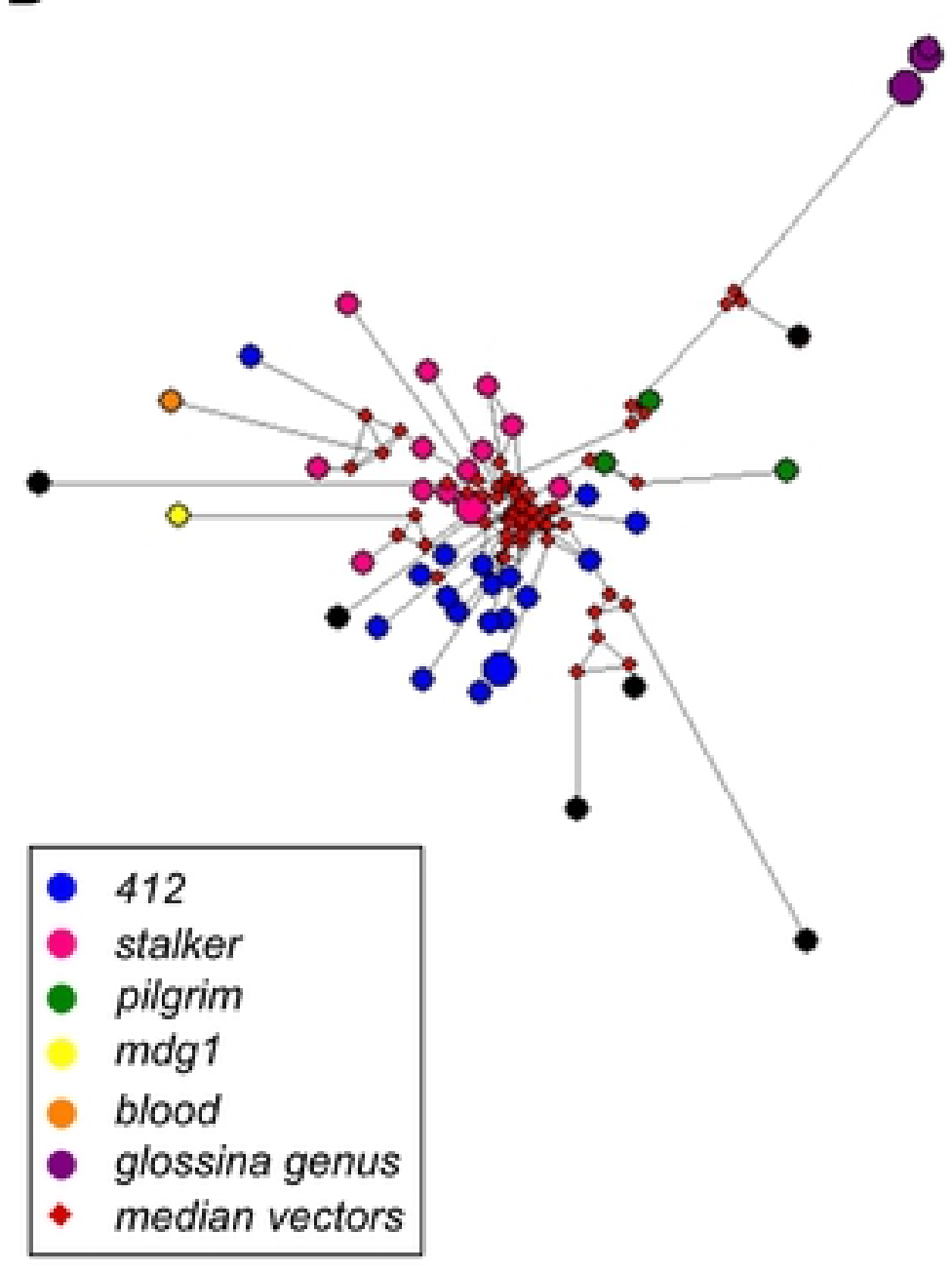

C

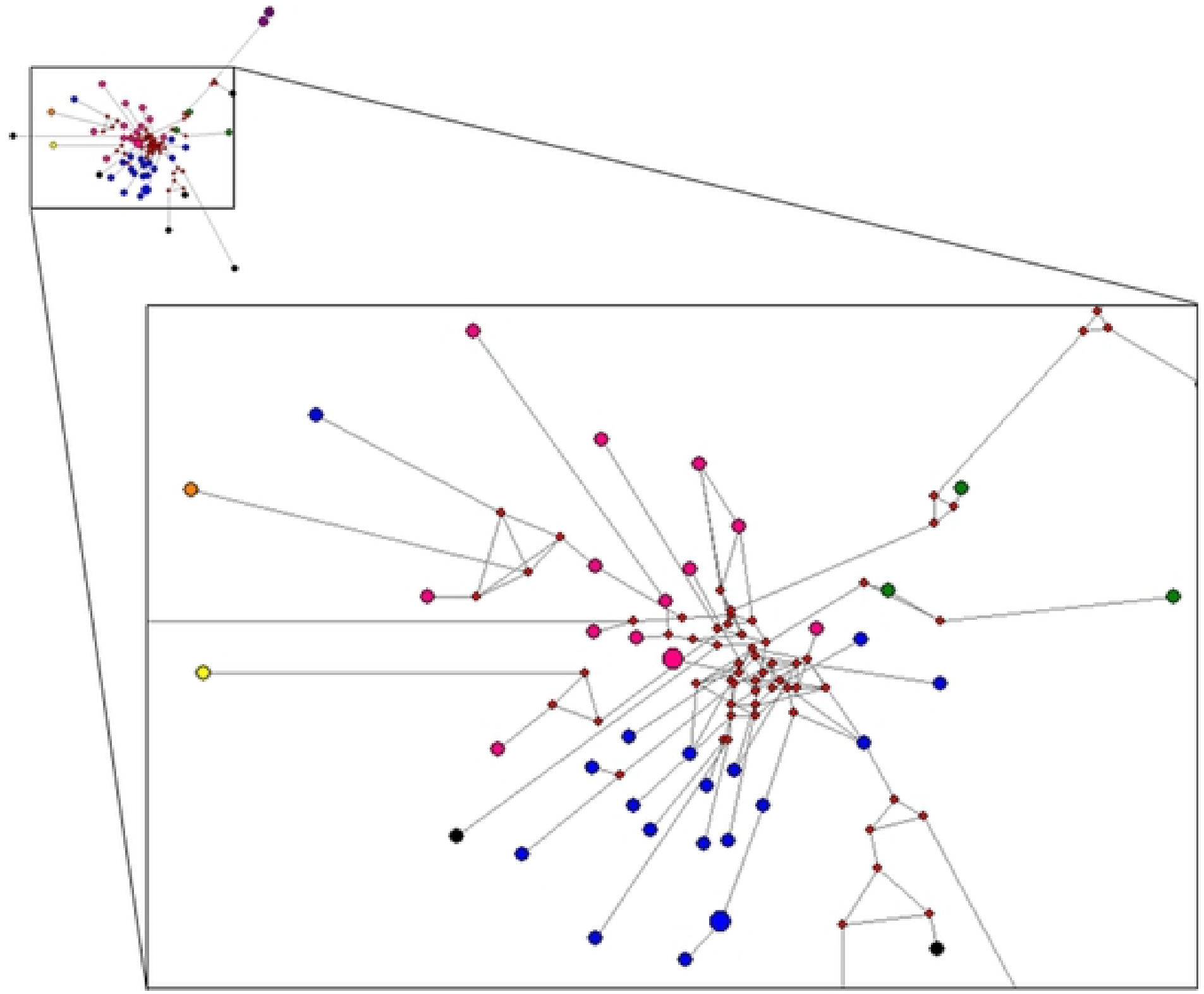

Figure 3 